\title{
Continuous dependence solutions for Hilfer fractional dif- ferential equations with nonlocal conditions
}

\section{Mohamed I. Abbas}

Department of Mathematics and Computer Science, Faculty of Science, Alexandria University, Alexandria 21511, Egypt.

\begin{abstract}
We discuss the existence, uniqueness and continuous dependence of solution for a non-autonomous semilinear Hilfer fractional differential equation with nonlocal conditions in the space of weighted continuous functions. By means of the Krasnoselskii's fixed point theorem and the generalized Gronwall's inequality, we establish the desired results.
\end{abstract}

Keywords: Hilfer fractional derivative, Krasnoselskii's fixed point theorem, Gronwall's inequality.

2010 MSC: 26A33, 34A08, 34A12.

(C)2019 All rights reserved.

\section{Introduction}

The fractional differential equations with Hilfer (generalized Riemann-Liouville) fractional derivative have recently attracted the attention of some authors interested in fractional calculus (see $[1,2,9,16,20$, $21,25,26])$. On the other hand, autonomous and non-autonomous systems of Hilfer fractional differential inclusions and equations play a considerable role that can not be over looked in the recent published researches. In [12], $\mathrm{Gu}$ and Trujillo discussed existence of mild solution of evolution equation with Hilfer fractional derivative. In [10], Gou and Li discussed the existence of mild solutions for nonlinear fractional non-autonomous evolution equations of Sobolev type with delay, one can refer to papers $[4,19,27,28]$. The continuous dependence on the initial data has been considered by many authors. Ye et al. [29] investigated a generalized Gronwall's inequality with singularity and used it for studying the dependence of the solution on the order and the initial condition of the Riemann-Liouville fractional differential equation

$$
\left\{\begin{array}{l}
D^{\alpha} y(t)=f(t, y(t)), \quad 0<\alpha<1,0 \leqslant t<T<+\infty, \\
\left.D^{\alpha-1} y(t)\right|_{t=0^{+}}=\eta .
\end{array}\right.
$$

Recently, Dhaigude et al. [5] discussed the existence, uniqueness and continuous dependence results of more general Hilfer fractional differential equation of the form

$$
\begin{cases}D_{a^{+}}^{\alpha, \beta} y(x)=f(x, y), & n-1<\alpha<n, 0 \leqslant \beta \leqslant 1, a \leqslant x<b, \\ I_{a^{+}}^{n-\gamma} y(a)=b_{k}, & k=1,2, \ldots, n, n=-[-\alpha], \gamma=\alpha+\beta(n-\alpha) .\end{cases}
$$

Email address: miabbas77@gmail .com (Mohamed I. Abbas)

doi: $10.22436 /$ jnsa.012.09.02 
Motivated by the above aforementioned papers, we consider the following non-autonomous semilinear Hilfer fractional differential equation

$$
\begin{cases}D_{0^{+}}^{\alpha, \beta} u(t)=A(t) u(t)+f(t, u(t)), & t \in J:=[0, T], \\ \left.I_{0^{+}}^{1-\gamma} u(t)\right|_{t=0^{+}}=u_{0}+g(u), & \alpha \leqslant \gamma=\alpha+\beta-\alpha \beta<1,\end{cases}
$$

in a Banach space $X$, where $D_{0^{+}}^{\alpha, \beta}$ is the Hilfer (generalized Riemann-Liouville) fractional derivative of order $\alpha \in(0,1)$ and type $\beta \in[0,1]$ introduced by Hilfer (see, $[13-15]$ ), $\mathrm{I}_{0^{+}}^{1-\gamma}$ is the left-sided RiemannLiouville fractional integral of order $1-\gamma$ and $u_{0} \in X .\{A(t), t \in J\}$ is a closed linear operator defined on a dense domain $D(A)$ in $X$ into $X$ such that $D(A)$ is independent of $t$ and $A(t)$ generates an evolution operator $\mathrm{U}(\mathrm{t}, \mathrm{s}), 0 \leqslant s \leqslant \mathrm{t} \leqslant \mathrm{T}$ in the Banach space $\mathrm{X}$ and the functions $\mathrm{f}: \mathrm{J} \times \mathrm{X} \rightarrow \mathrm{X}, \mathrm{g}: \mathrm{C}_{1-\gamma}(\mathrm{J}, \mathrm{X}) \rightarrow \mathrm{X}$ satisfy some assumptions that will be specified later.

It would be useful to emphasize that to the best of our knowledge, the results we obtained are more general than previous results, since the antecedent authors did not work on the operator $A(t)$ as well as the concept of mild solution in terms of the evolution operator $U(t, s)$ in the treatment of Hilfer fractional differential equations.

The rest of the paper is organized as follows. In Section 2, some preliminary results and notations are provided. The existence and uniqueness results are proved in Section 3. The dependence of solutions on order and initial conditions is studied in the last section.

\section{Preliminaries}

In this section we present some definitions and lemmas which will be used in our results later. At first, we review some fundamental definitions of the Riemann-Liouville fractional integral and derivative which will be made up to the Hilfer fractional derivative (see $[9,17])$.

Definition 2.1. The left-sided Riemann-Liouville fractional integral of order $\alpha>0$ of a function $y$ : $[0,+\infty) \rightarrow \mathbb{R}$ is defined as

$$
\mathrm{I}_{0^{+}}^{\alpha} \mathrm{y}(\mathrm{t})=\frac{1}{\Gamma(\alpha)} \int_{0}^{\mathrm{t}}(\mathrm{t}-\mathrm{s})^{\alpha-1} \mathrm{y}(\mathrm{s}) \mathrm{ds}, \mathrm{t}>0,
$$

where $\Gamma(\cdot)$ is the Gamma function.

Definition 2.2. The left-sided Riemann-Liouville fractional derivative of order $\alpha \in[n-1, n), n \in \mathbb{Z}^{+}$of a function $y:[0,+\infty) \rightarrow \mathbb{R}$ is defined as

$$
D_{0^{+}}^{\alpha} y(t)=\frac{1}{\Gamma(n-\alpha)}\left(\frac{d}{d t}\right)^{n} \int_{0}^{t}(t-s)^{n-\alpha-1} y(s) d s, t>0 .
$$

Definition 2.3 (Hilfer fractional derivative). The right-sided Hilfer fractional derivative operator of order $0<\alpha<1$ and type $0 \leqslant \beta \leqslant 1$ is defined by

$$
\mathrm{D}_{0^{+}}^{\alpha, \beta} \mathrm{y}(\mathrm{t})=\left(\mathrm{I}_{0^{+}}^{\beta(1-\alpha)} \mathrm{D}\left(\mathrm{I}_{0^{+}}^{(1-\beta)(1-\alpha)} \mathrm{y}\right)\right)(\mathrm{t}),
$$

where $D:=\frac{d}{d t}$.

This generalization (2.1) yields the classical Riemann-Liouville fractional derivative operator when $\beta=0$. Moreover, for $\beta=1$, it gives the Caputo fractional derivative operator.

In view of the Laplace transform of the Hilfer derivative ([24], formula (1.6))

$$
\mathcal{L}\left[D_{0^{+}}^{\alpha, \beta} y\right](s)=s^{\alpha} \mathcal{L}[y](s)-s^{\beta(\alpha-1)}\left(I_{0^{+}}^{(1-\alpha)(1-\beta)} y\right)\left(0^{+}\right),
$$


it is clear that the initial conditions that must be considered are of the form $\left(\mathrm{I}_{0^{+}}^{(1-\alpha)(1-\beta)} \mathrm{y}\right)\left(0^{+}\right)$, i.e., on the initial value of the fractional integral of order $1-\gamma=(1-\alpha)(1-\beta)$.

Some properties and applications of the generalized Riemann-Liouville fractional derivative are given in [13].

Let $\mathrm{C}(\mathrm{J}, \mathrm{X})$ be the Banach space of all $X$-valued continuous functions from $J$ into $X$ equipped by the norm $\|x\|_{C}=\sup _{t \in J}|x(t)|, \forall x \in C(J, X)$. For $0 \leqslant \gamma<1$, we define the weighted space $C_{1-\gamma}(J, X)$ of all $X$-valued continuous functions $h$ as

$$
C_{1-\gamma}(J, X)=\left\{h: J \rightarrow X: t^{1-\gamma} h(t) \in C(J, X)\right\},
$$

with the norm

$$
\|\mathrm{h}\|_{\mathrm{e}_{1-\gamma}}=\left\|\mathrm{t}^{1-\gamma} \mathrm{h}(\mathrm{t})\right\|_{\mathrm{C}} .
$$

Evidently, $\mathrm{C}_{1-\gamma}(\mathrm{J}, \mathrm{X})$ is a Banach space.

In order to solve our problem, the following weighted spaces are presented

$$
C_{1-\gamma}^{\alpha, \beta}(J, X)=\left\{h \in C_{1-\gamma}(J, X), D_{0^{+}}^{\alpha, \beta} h(t) \in C_{1-\gamma}(J, X)\right\}
$$

and

$$
\mathrm{C}_{1-\gamma}^{\gamma}(\mathrm{J}, \mathrm{X})=\left\{\mathrm{h} \in \mathrm{C}_{1-\gamma}(\mathrm{J}, \mathrm{X}), \mathrm{D}_{0^{+}}^{\gamma} \mathrm{h}(\mathrm{t}) \in \mathrm{C}_{1-\gamma}(\mathrm{J}, \mathrm{X})\right\} .
$$

It is clear that $\mathrm{C}_{1-\gamma}^{\gamma}(\mathrm{J}, \mathrm{X}) \subset \mathrm{C}_{1-\gamma}^{\alpha, \beta}(\mathrm{J}, \mathrm{X})$.

Definition $2.4([6-8,23])$. The family $\{A(t), t \in J\}$ generates a unique linear evolution operator $U(t, s), 0 \leqslant$ $s \leqslant t \leqslant T$ satisfying the following properties:

(a) $\mathrm{U}(\mathrm{t}, \mathrm{s}) \in \mathrm{L}(\mathrm{X})$, the space of all linear transformations on $X$, whenever $0 \leqslant s \leqslant t \leqslant T$ and for each $x \in X$, the mapping $(t, s) \rightarrow U(t, s) x$ is continuous;

(b) $\mathrm{U}(\mathrm{t}, \mathrm{s}) \mathrm{U}(\mathrm{s}, \tau)=\mathrm{U}(\mathrm{s}, \tau)$ for $0 \leqslant \tau \leqslant s \leqslant \mathrm{t} \leqslant \mathrm{T}$;

(c) $\mathrm{U}(\mathrm{t}, \mathrm{t})=\mathrm{I}$ (identity);

(d) $\mathrm{U}(\mathrm{t}, \mathrm{s})$ is a compact operator whenever $\mathrm{t}-\mathrm{s}>0$;

(e) $\frac{\partial \mathrm{U}}{\partial t}(t, s) x=-A(t) U(t, s)$ for $s<t$;

(f) there is a constant $M_{1} \geqslant 1$ such that $\|\mathrm{U}(\mathrm{t}, \mathrm{s})\| \leqslant \mathrm{M}_{1}, 0 \leqslant \mathrm{~s} \leqslant \mathrm{t} \leqslant \mathrm{T}$;

(g) if $0<\mathrm{h}<1, \mathrm{t}-\mathrm{s}>\mathrm{h}$, and $0<v<1$, then

$$
\|\mathrm{U}(\mathrm{t}+\mathrm{h}, \mathrm{s})-\mathrm{U}(\mathrm{t}, \mathrm{s})\| \leqslant \frac{\mathrm{M}_{2} \mathrm{~h}^{v}}{|t-s|^{v}}
$$

for some $\mathrm{M}_{2}>0$;

(h) if $f(t)$ is continuous on $[0, T]$, then the function $t \rightarrow \int_{0}^{t} U(t, s) f(s) d s$ is Hölder continuous with any exponent $0<v<1$.

In order to define the concept of mild solution for the non-autonomous Hilfer fractional differential equation (1.1), by comparison with the previous results in the case of Caputo derivative $([3,18,22])$ and those in the case of Hilfer derivative [9], we obtain the following definition immediately.

Definition 2.5. A function $u \in C_{1-\gamma}(J, X)$ is said to be a mild solution of problem (1.1) if $\left.I_{0^{+}}^{1-\gamma} \mathfrak{u}(\mathfrak{t})\right|_{t=0^{+}}=$ $u_{0}+g(u)$, then $u$ satisfies the integral equation

$$
u(t)=u(t, 0) \frac{\left(u_{0}+g(u)\right)}{\Gamma(\gamma)} t^{\gamma-1}+\frac{1}{\Gamma(\alpha)} \int_{0}^{t}(t-s)^{\alpha-1} u(t, s) f(s, u(s)) d s
$$

Theorem 2.6 ([11, Krasnoselskii's fixed point theorem]). Let $\Omega$ be a closed convex and non-empty subset of a Banach space $(X,\|\cdot\|)$. Suppose that $\mathcal{A}$ and $\mathcal{B}$ maps $\Omega$ into $X$, such that the following hypotheses are fulfilled:

(i) $\mathcal{A x}+\mathcal{B} y \in \Omega$ for all $x, y \in \Omega$;

(ii) $\mathcal{A}$ is a contraction mapping;

(iii) $\mathcal{B}$ is compact and continuous.

Then there exists a $z \in \Omega$ such that $z=\mathcal{A z}+\mathcal{B} z$. 


\section{Existence and uniqueness results}

In this section we prove the existence and uniqueness of solution for the problem (1.1). At beginning, we give the following assumptions.

(H1) For each $t \in J$, the function $f(t, \cdot): X \rightarrow X$ is continuous and for each $x \in X$, the function $f(\cdot, x): J \rightarrow$ $\mathrm{X}$ is strongly measurable.

(H2) There exist two constants $\mathrm{L}_{1}, \mathrm{~L}_{2}>0$ such that

$$
|f(t, u)-f(t, v)| \leqslant L_{1}|u-v| \text { for each } t \in J, u, v \in X,
$$

and

$$
\mathrm{L}_{2}=\sup _{\mathrm{t} \in \mathrm{J}}|\mathrm{f}(\mathrm{t}, 0)| .
$$

(H3) $g: \mathrm{C}_{1-\gamma}(\mathrm{J}, \mathrm{X}) \rightarrow \mathrm{X}$ is a continuous function and there exist two constants $\mathrm{L}_{3}, \mathrm{~L}_{4}>0$ such that

$$
\left|g\left(u_{1}\right)-g\left(u_{2}\right)\right| \leqslant L_{3}\left\|u_{1}-u_{2}\right\|_{C_{1-\gamma}} \text { for all } u_{1}, u_{2} \in C_{1-\gamma}(J, X), \quad L_{4}=|g(0)| .
$$

The following uniqueness result is based on the Banach contraction principle.

Theorem 3.1. Assume that assumptions (H1)-(H3) hold. Then the problem (1.1) has a unique solution $\mathrm{u} \in$ $\mathrm{C}_{1-\gamma}(\mathrm{J}, \mathrm{X})$ if

$$
M_{1}\left(\frac{\mathrm{L}_{3}}{\Gamma(\gamma)}+\frac{\mathrm{L}_{1} \mathrm{~T}^{1-\gamma+\alpha}}{\Gamma(\alpha+1)}\right)<1
$$

Proof. Define the operator $\mathcal{F}: \mathrm{C}_{1-\gamma}(\mathrm{J}, \mathrm{X}) \rightarrow \mathrm{C}_{1-\gamma}(\mathrm{J}, \mathrm{X})$ by

$$
(\mathcal{F u})(\mathrm{t})=\frac{\left(\mathrm{u}_{0}+\mathrm{g}(\mathrm{u})\right) \mathrm{t}^{\gamma-1}}{\Gamma(\gamma)} \mathrm{u}(\mathrm{t}, 0)+\frac{1}{\Gamma(\alpha)} \int_{0}^{t}(t-s)^{\alpha-1} \mathrm{u}(\mathrm{t}, \mathrm{s}) \mathrm{f}(\mathrm{s}, \mathrm{u}(\mathrm{s})) \mathrm{ds}
$$

From Definition 2.5, it is easy to verify that the mild solution of the problem (1.1) is equivalent to the fixed point of the operator $\mathcal{F}$. For any $u, v \in \mathrm{C}_{1-\gamma}(\mathrm{J}, \mathrm{X})$ and for each $\mathrm{t} \in \mathrm{J}$, we get

$$
\begin{aligned}
& \left|t^{1-\gamma}(\mathcal{F u})(t)-t^{1-\gamma}(\mathcal{F v})(t)\right|=\mid \frac{(g(u)-g(v))}{\Gamma(\gamma)} \mathrm{U}(\mathrm{t}, 0)+\frac{t^{1-\gamma}}{\Gamma(\alpha)} \int_{0}^{t}(t-s)^{\alpha-1} \mathrm{u}(\mathrm{t}, \mathrm{s})(f(s, u(s))-f(s, v(s)) d s \mid \\
& \leqslant \frac{|g(u)-g(v)|}{\Gamma(\gamma)}|\mathrm{u}(\mathrm{t}, 0)|+\frac{\mathrm{t}^{1-\gamma}}{\Gamma(\alpha)} \int_{0}^{\mathrm{t}}(\mathrm{t}-\mathrm{s})^{\alpha-1}|\mathrm{U}(\mathrm{t}, \mathrm{s})||\mathrm{f}(\mathrm{s}, \mathrm{u}(\mathrm{s}))-\mathrm{f}(\mathrm{s}, v(\mathrm{~s}))| \mathrm{ds} \\
& \leqslant \frac{M_{1} L_{3}}{\Gamma(\gamma)}\|u-v\|_{C_{1-\gamma}}+\frac{M_{1} L_{1} t^{1-\gamma}}{\Gamma(\alpha)} \int_{0}^{t}(t-s)^{\alpha-1}|u(s)-v(s)| d s \\
& \leqslant M_{1}\left(\frac{\mathrm{L}_{3}}{\Gamma(\gamma)}+\frac{\mathrm{L}_{1} \mathrm{~T}^{1-\gamma+\alpha}}{\Gamma(\alpha+1)}\right)\|u-v\|_{\mathrm{C}_{1-\gamma}} \text {. }
\end{aligned}
$$

Thus

$$
\|\mathcal{F u}-\mathcal{F} v\|_{c_{1-\gamma}} \leqslant M_{1}\left(\frac{L_{3}}{\Gamma(\gamma)}+\frac{L_{1} T^{1-\gamma+\alpha}}{\Gamma(\alpha+1)}\right)\|u-v\|_{c_{1-\gamma}}
$$

which by (3.1) implies that $\mathcal{F}$ is a contraction mapping. It follows that $\mathcal{F}$ has a unique fixed point, which is a solution of the problem (1.1).

Our next result is based on the Krasnoselskii's the fixed point theorem.

Theorem 3.2. Under the assumptions (H1)-(H3), the problem (1.1) has at least one solution on $\mathrm{J}$ if $\frac{\mathrm{M}_{1} \mathrm{~L}_{3}}{\Gamma(\gamma)}<1$. 
Proof. Let $\mathrm{B}_{\mathrm{r}}=\left\{x \in \mathrm{C}_{1-\gamma}(\mathrm{J}, \mathrm{X}):\|x\|_{\mathrm{C}_{1-\gamma}} \leqslant \mathrm{r}\right\}$ with

$$
r \geqslant \frac{M_{1}\left(\frac{\left|\mathfrak{u}_{0}\right|+\mathrm{L}_{4}}{\Gamma(\gamma)}+\frac{\mathrm{L}_{2} T^{1-\gamma+\alpha}}{\Gamma(\alpha+1)}\right)}{1-M_{1}\left(\frac{\mathrm{L}_{3}}{\Gamma(\gamma)}+\frac{\mathrm{L}_{1} T^{1-\gamma+\alpha}}{\Gamma(\alpha+1)}\right)} .
$$

Then $B_{r}$ is a closed, bounded and convex subset of $C_{1-\gamma}(J, X)$. We define the operators $\mathcal{A}$ and $\mathcal{B}$ as follows

$$
(\mathcal{A u})(\mathrm{t})=\frac{\left(\mathrm{u}_{0}+\mathrm{g}(\mathrm{u})\right) \mathrm{t}^{\gamma-1}}{\Gamma(\gamma)} \mathrm{u}(\mathrm{t}, 0)
$$

and

$$
(\mathcal{B u})(t)=\frac{1}{\Gamma(\alpha)} \int_{0}^{t}(t-s)^{\alpha-1} u(t, s) f(s, u(s)) d s .
$$

We show that $\mathcal{A}+\mathcal{B}$ has a fixed point in $\mathrm{B}_{\mathrm{r}}$. The proof is divided into three steps.

Step1. We show that $\mathcal{A} u+\mathcal{B} v \in \mathrm{B}_{\mathrm{r}}$. For every $u, v \in \mathrm{B}_{\mathrm{r}}$ and each $\mathrm{t} \in \mathrm{J}$, we get

$$
\begin{aligned}
\left|t^{1-\gamma}(\mathcal{A u})(\mathrm{t})+\mathrm{t}^{1-\gamma}(\mathcal{B} v)(\mathrm{t})\right|= & \left|\frac{\left(\mathrm{u}_{0}+\mathrm{g}(\mathrm{u})\right)}{\Gamma(\gamma)} \mathrm{U}(\mathrm{t}, 0)+\frac{\mathrm{t}^{1-\gamma}}{\Gamma(\alpha)} \int_{0}^{\mathrm{t}}(\mathrm{t}-\mathrm{s})^{\alpha-1} \mathrm{u}(\mathrm{t}, \mathrm{s}) \mathrm{f}(\mathrm{s}, \mathrm{v}(\mathrm{s})) \mathrm{ds}\right| \\
\leqslant & \frac{\left(\left|\mathrm{u}_{0}\right|+|\mathrm{g}(\mathrm{u})-\mathrm{g}(0)|+|\mathrm{g}(0)|\right)}{\Gamma(\gamma)}|\mathrm{U}(\mathrm{t}, 0)| \\
& +\frac{\mathrm{t}^{1-\gamma}}{\Gamma(\alpha)} \int_{0}^{\mathrm{t}}(\mathrm{t}-\mathrm{s})^{\alpha-1}|\mathrm{U}(\mathrm{t}, \mathrm{s})|(|\mathrm{f}(\mathrm{s}, v(\mathrm{~s}))-\mathrm{f}(\mathrm{s}, 0)|+|\mathrm{f}(\mathrm{s}, 0)|) \mathrm{d} s \\
\leqslant & \frac{\mathrm{M}_{1}\left(\left|\mathrm{u}_{0}\right|+\mathrm{L}_{3} r+\mathrm{L}_{4}\right)}{\Gamma(\gamma)}+\frac{\mathrm{M}_{1} \mathrm{t}^{1-\gamma}}{\Gamma(\alpha)}\left(\mathrm{L}_{1} \mathrm{r}+\mathrm{L}_{2}\right) \int_{0}^{\mathrm{t}}(\mathrm{t}-\mathrm{s})^{\alpha-1} \mathrm{ds} \\
\leqslant & \mathrm{M}_{1}\left(\frac{\left|\mathrm{u}_{0}\right|+\mathrm{L}_{4}}{\Gamma(\gamma)}+\frac{\mathrm{L}_{2} \mathrm{~T}^{1-\gamma+\alpha}}{\Gamma(\alpha+1)}\right)+\mathrm{M}_{1}\left(\frac{\mathrm{L}_{3}}{\Gamma(\gamma)}+\frac{\mathrm{L}_{1} \mathrm{~T}^{1-\gamma+\alpha}}{\Gamma(\alpha+1)}\right) \mathrm{r} \leqslant \mathrm{r} .
\end{aligned}
$$

Therefore,

$$
\|\mathcal{A} u+\mathcal{B} v\|_{\mathrm{C}_{1-\gamma}} \leqslant \mathrm{r},
$$

which implies that $\mathcal{A} u+\mathcal{B} v \in \mathrm{B}_{\mathrm{r}}$ for every $u, v \in \mathrm{B}_{\mathrm{r}}$.

Step2. The operator $\mathcal{A}$ is contraction on $B_{r}$. For each $u, v \in B_{r}$ and each $t \in J$, we get

$$
\left|t^{1-\gamma}(\mathcal{A u})(t)-t^{1-\gamma}(\mathcal{A v})(t)\right| \leqslant \frac{\mid g(u)-g(v)}{\Gamma(\gamma) \mid}|\mathrm{u}(\mathrm{t}, 0)| \leqslant \frac{M_{1} \mathrm{~L}_{3}}{\Gamma(\gamma)}\|\mathrm{u}-v\|_{\mathrm{C}_{1-\gamma}} .
$$

Then

$$
\|\mathcal{A u}-\mathcal{A} v\|_{\mathrm{C}_{1-\gamma}} \leqslant \frac{\mathrm{M}_{1} \mathrm{~L}_{3}}{\Gamma(\gamma)}\|\mathrm{u}-v\|_{\mathrm{C}_{1-\gamma}}
$$

with $\frac{M_{1} L_{3}}{\Gamma(\gamma)}<1$. This shows that $\mathcal{A}$ is contraction on $B_{r}$.

Step3. The operator $\mathcal{B}$ is compact and continuous. We know that

$$
\left|t^{1-\gamma}(\mathcal{B} x)(t)\right| \leqslant \frac{t^{1-\gamma}}{\Gamma(\alpha)} \int_{0}^{t}(t-s)^{\alpha-1}|U(t, s)|(|f(s, v(s))-f(s, 0)|+|f(s, 0)|) d s \leqslant \frac{M_{1} T^{1-\gamma+\alpha}}{\Gamma(\alpha+1)}\left(L_{1} r+L_{2}\right) .
$$

Thus $\|\mathcal{B} u\|_{C_{1-\gamma}} \leqslant \frac{M_{1} T^{1-\gamma+\alpha}}{\Gamma(\alpha+1)}\left(L_{1} r+L_{2}\right)$, this shows that $\mathcal{B}$ is uniformly bounded on $B_{r}$. 
It remains to prove the compactness of operator $\mathcal{B}$. For $t_{1}, t_{2} \in J, t_{1}<t_{2}$, we have

$$
\begin{aligned}
&\left|t_{2}^{1-\gamma}(\mathcal{B u})\left(t_{2}\right)-t_{1}^{1-\gamma}(\mathcal{B u})\left(t_{1}\right)\right| \\
&=\left|\frac{t_{2}^{1-\gamma}}{\Gamma(\alpha)} \int_{0}^{t_{2}}\left(t_{2}-s\right)^{\alpha-1} u\left(t_{2}, s\right) f(s, u(s)) d s-\frac{t_{1}^{1-\gamma}}{\Gamma(\alpha)} \int_{0}^{t_{1}}\left(t_{1}-s\right)^{\alpha-1} u\left(t_{1}, s\right) f(s, u(s)) d s\right| \\
& \leqslant\left|\frac{1}{\Gamma(\alpha)} \int_{t_{1}}^{t_{2}} t_{2}^{1-\gamma}\left(t_{2}-s\right)^{\alpha-1} u\left(t_{2}, s\right) f(s, u(s)) d s\right| \\
&+\left|\frac{1}{\Gamma(\alpha)} \int_{0}^{t_{1}}\left(t_{2}^{1-\gamma}\left(t_{2}-s\right)^{\alpha-1}-t_{1}^{1-\gamma}\left(t_{1}-s\right)^{\alpha-1}\right) u\left(t_{2}, s\right) f(s, u(s)) d s\right| \\
&+\left|\frac{1}{\Gamma(\alpha)} \int_{0}^{t_{1}} t_{1}^{1-\gamma}\left(t_{1}-s\right)^{\alpha-1}\left(u\left(t_{2}, s\right)-u\left(t_{1}, s\right)\right) f(s, u(s)) d s\right| \\
&= I_{1}+I_{2}+I_{3},
\end{aligned}
$$

where

$$
\begin{aligned}
& I_{1}=\left|\frac{1}{\Gamma(\alpha)} \int_{t_{1}}^{t_{2}} t_{2}^{1-\gamma}\left(t_{2}-s\right)^{\alpha-1} u\left(t_{2}, s\right) f(s, u(s)) d s\right|, \\
& I_{2}=\left|\frac{1}{\Gamma(\alpha)} \int_{0}^{t_{1}}\left(t_{2}^{1-\gamma}\left(t_{2}-s\right)^{\alpha-1}-t_{1}^{1-\gamma}\left(t_{1}-s\right)^{\alpha-1}\right) U\left(t_{2}, s\right) f(s, u(s)) d s\right|, \\
& I_{3}=\left|\frac{1}{\Gamma(\alpha)} \int_{0}^{t_{1}} t_{1}^{1-\gamma}\left(t_{1}-s\right)^{\alpha-1}\left(U\left(t_{2}, s\right)-U\left(t_{1}, s\right)\right) f(s, u(s)) d s\right| .
\end{aligned}
$$

By assumptions (H1)-(H3) together with Definition 2.4, we get

$$
\begin{aligned}
& I_{1} \leqslant \frac{M_{1}\left(L_{1} r+L_{2}\right)}{\Gamma(\alpha+1)} t_{2}^{1-\gamma}\left(t_{2}-t_{1}\right)^{\alpha}, \\
& I_{2} \leqslant \frac{M_{1}\left(L_{1} r+L_{2}\right)}{\Gamma(\alpha+1)}\left(\left(t_{2}^{1-\gamma+\alpha}-t_{1}^{1-\gamma+\alpha}\right)-t_{2}^{1-\gamma}\left(t_{2}-t_{1}\right)^{\alpha}\right), \\
& I_{3} \leqslant \frac{M_{2}\left(L_{1} r+L_{2}\right)}{(\alpha-v) \Gamma(\alpha)} t_{1}^{1-\gamma+\alpha-v}\left(t_{2}-t_{1}\right)^{v} .
\end{aligned}
$$

By substitution in (3.2), we have

$$
\left\|(\mathcal{B u})\left(t_{2}\right)-(\mathcal{B u})\left(t_{1}\right)\right\|_{C_{1-\gamma}} \leqslant \frac{M_{1}\left(L_{1} r+L_{2}\right)}{\Gamma(\alpha+1)}\left(t_{2}^{1-\gamma+\alpha}-t_{1}^{1-\gamma+\alpha}\right)+\frac{M_{2}\left(L_{1} r+L_{2}\right)}{(\alpha-v) \Gamma(\alpha)} t_{1}^{1-\gamma+\alpha-v}\left(t_{2}-t_{1}\right)^{v},
$$

As $t_{2} \rightarrow t_{1}$, the right-hand side of the above inequality tends to zero. Thus, $\mathcal{B}$ is equicontinuous.

As a consequence of Steps 1-3 together with the Arzelà-Ascoli theorem, we deduce that the operator $\mathcal{B}$ is compact and continuous. Finally, By the Krasnoskelskii's fixed point theorem, we have the conclusion of the theorem.

\section{Continuous dependence of mild solution}

In this section we study the continuous dependence of solution of the Hilfer fractional differential equation (1.1) on the order and the initial condition using generalized Gronwall's inequality [29].

Theorem 4.1. Let $\alpha>0$ and $\delta>0$ such that $0<\alpha-\delta<\alpha \leqslant 1$. Let $\mathrm{f}$ be a continuous function such that the assumption $(\mathrm{H} 2)$ is satisfied. For $0 \leqslant \mathrm{t} \leqslant \mathrm{b}<\mathrm{T}$, assume that $\mathrm{u}$ and $\widetilde{\mathrm{u}}$ are the solutions of the problem (1.1) and

$$
\begin{cases}D_{0^{+}}^{\alpha-\delta, \beta} \widetilde{u}(t)=A(t) \widetilde{u}(t)+f(t, \widetilde{u}(t)), & t \in J=[0, T], \\ \left.I_{0^{+}}^{1-\gamma-\delta(\beta-1)} \widetilde{u}(t)\right|_{t=0^{+}}=\widetilde{u}_{0}+g(\widetilde{u}), & \alpha \leqslant \gamma=\alpha+\beta-\alpha \beta<1,\end{cases}
$$


respectively. Then for $0 \leqslant \mathrm{t} \leqslant \mathrm{b}<\mathrm{T}$ the following inequality holds

$$
|\widetilde{u}(t)-u(t)| \leqslant \Omega(t)+\int_{0}^{t} \frac{(K \Gamma(\alpha-\delta))^{n}}{\Gamma(n(\alpha-\delta))}(t-s)^{n(\alpha-\delta)-1} \Omega(s) d s,
$$

where

$$
\begin{aligned}
\Omega(t)= & M_{1}\left|\frac{\left(\widetilde{u}_{0}+g(\widetilde{u})\right)}{\Gamma(\gamma+\delta(\beta-1))} t^{\gamma+\delta(\beta-1)-1}-\frac{\left(u_{0}+g(u)\right)}{\Gamma(\gamma)} t^{\gamma-1}\right| \\
& +M_{1}\|f\|\left|\frac{t^{\alpha-\delta}}{\Gamma(\alpha-\delta+1)}-\frac{t^{\alpha-\delta}}{(\alpha-\delta) \Gamma(\alpha)}\right|+M_{1}\|f\|\left|\frac{t^{\alpha-\delta}}{(\alpha-\delta) \Gamma(\alpha)}-\frac{t^{\alpha}}{\Gamma(\alpha+1)}\right|, \\
K= & \frac{M_{1} L_{1}}{\Gamma(\alpha)}, \text { and }\|f\|=\max _{0 \leqslant t \leqslant b}|f(t, u(t))| .
\end{aligned}
$$

Proof. The problems (1.1) and (4.1) are equivalent to the following integral equations

$$
u(t)=u(t, 0) \frac{\left(u_{0}+g(u)\right)}{\Gamma(\gamma)} t^{\gamma-1}+\frac{1}{\Gamma(\alpha)} \int_{0}^{t}(t-s)^{\alpha-1} u(t, s) f(s, u(s)) d s,
$$

and

$$
\widetilde{u}(t)=u(t, 0) \frac{\left(\widetilde{u}_{0}+g(\widetilde{u})\right)}{\Gamma(\gamma+\delta(\beta-1))} t^{\gamma+\delta(\beta-1)-1}+\frac{1}{\Gamma(\alpha-\delta)} \int_{0}^{t}(t-s)^{\alpha-\delta-1} u(t, s) f(s, \widetilde{u}(s)) d s,
$$

respectively for each $t \in J$. Then we have

$$
\begin{aligned}
& |\widetilde{u}(t)-u(t)| \leqslant\left|\left(\frac{\left(\widetilde{u}_{0}+g(\widetilde{u})\right)}{\Gamma(\gamma+\delta(\beta-1))} t^{\gamma+\delta(\beta-1)-1}-\frac{\left(u_{0}+g(u)\right)}{\Gamma(\gamma)} t^{\gamma-1}\right) u(t, 0)\right| \\
& +\left|\int_{0}^{t} \frac{(t-s)^{\alpha-\delta-1}}{\Gamma(\alpha-\delta)} u(t, s) f(s, \widetilde{u}(s)) d s-\int_{0}^{t} \frac{(t-s)^{\alpha-1}}{\Gamma(\alpha)} u(t, s) f(s, u(s)) d s\right| \\
& \leqslant\left|\left(\frac{\left(\widetilde{u}_{0}+g(\widetilde{u})\right)}{\Gamma(\gamma+\delta(\beta-1))} t^{\gamma+\delta(\beta-1)-1}-\frac{\left(\mathrm{u}_{0}+\mathrm{g}(\mathrm{u})\right)}{\Gamma(\gamma)} \mathrm{t}^{\gamma-1}\right) \mathrm{u}(\mathrm{t}, 0)\right| \\
& +\left|\int_{0}^{t}\left(\frac{(t-s)^{\alpha-\delta-1}}{\Gamma(\alpha-\delta)}-\frac{(t-s)^{\alpha-\delta-1}}{\Gamma(\alpha)}\right) u(t, s) f(s, \widetilde{u}(s)) d s\right| \\
& +\left|\int_{0}^{t}\left(\frac{(t-s)^{\alpha-\delta-1}}{\Gamma(\alpha)}-\frac{(t-s)^{\alpha-1}}{\Gamma(\alpha)}\right) u(t, s) f(s, u(s)) d s\right| \\
& +\left|\int_{0}^{t} \frac{(t-s)^{\alpha-\delta-1}}{\Gamma(\alpha)} u(t, s)(f(s, \widetilde{u}(s))-f(s, u(s))) d s\right| \\
& \leqslant M_{1}\left|\frac{\left(\widetilde{u}_{0}+g(\widetilde{u})\right)}{\Gamma(\gamma+\delta(\beta-1))} t^{\gamma+\delta(\beta-1)-1}-\frac{\left(u_{0}+g(u)\right)}{\Gamma(\gamma)} t^{\gamma-1}\right| \\
& +M_{1}\|f\|\left|\frac{t^{\alpha-\delta}}{\Gamma(\alpha-\delta+1)}-\frac{t^{\alpha-\delta}}{(\alpha-\delta) \Gamma(\alpha)}\right|+M_{1}\|f\|\left|\frac{t^{\alpha-\delta}}{(\alpha-\delta) \Gamma(\alpha)}-\frac{t^{\alpha}}{\Gamma(\alpha+1)}\right| \\
& +\frac{\mathrm{M}_{1} \mathrm{~L}_{1}}{\Gamma(\alpha)} \int_{0}^{\mathrm{t}}(\mathrm{t}-\mathrm{s})^{\alpha-\delta-1}|\widetilde{\mathrm{u}}(\mathrm{s})-\mathrm{u}(\mathrm{s})| \mathrm{ds},
\end{aligned}
$$

this implies that

$$
|\widetilde{\mathfrak{u}}(\mathrm{t})-\mathfrak{u}(\mathrm{t})| \leqslant \Omega(t)+K \int_{0}^{t}(t-s)^{\alpha-\delta-1}|\widetilde{\mathfrak{u}}(s)-\mathfrak{u}(s)| d s .
$$

Using the generalized Gronwall's inequality ([29, Corollary 1]), we get

$$
|\widetilde{u}(t)-u(t)| \leqslant \Omega(t)+\int_{0}^{t} \frac{(K \Gamma(\alpha-\delta))^{n}}{\Gamma(n(\alpha-\delta))}(t-s)^{n(\alpha-\delta)-1} \Omega(s) d s .
$$


Next, we consider the Hilfer fractional differential equation

$$
\begin{cases}D_{0^{+}}^{\alpha, \beta} \widehat{u}(t)=A(t) \widehat{u}(t)+f(t, \widehat{u}(t)), & t \in J:=[0, T], \\ \left.I_{0^{+}}^{1-} \widehat{u}(t)\right|_{t=0^{+}}=\widehat{u}_{0}+g(\widehat{u}), & \alpha \leqslant \gamma=\alpha+\beta-\alpha \beta<1 .\end{cases}
$$

Theorem 4.2. Suppose that the assumptions of Theorems 3.1 and 3.2 hold. If $u(t)$ is a solution of the problem (1.1) and $\widehat{u}(t)$ is a solution of the problem (4.2). Then the following relation is satisfied

$$
\|\widehat{u}-u\|_{c_{1-\gamma}} \leqslant \frac{\frac{M_{1}}{\Gamma(\gamma)}}{1-M_{1}\left(\frac{L_{3}}{\Gamma(\gamma)}+\frac{L_{1} T^{1-\gamma+\alpha}}{\Gamma(\alpha+1)}\right)}\left|\widehat{u}_{0}-u_{0}\right| .
$$

Proof. For each $t \in \mathrm{J}$, we have

$$
\begin{aligned}
\left|t^{1-\gamma} \widehat{u}(t)-t^{1-\gamma} \mathfrak{u}(t)\right| \leqslant & \left|\frac{1}{\Gamma(\gamma)}\left(\left(\widehat{u}_{0}-u_{0}\right)+(g(\widehat{u})-g(u))\right) u(t, 0)\right| \\
& +\left|\frac{t^{1-\gamma}}{\Gamma(\alpha)} \int_{0}^{t}(t-s)^{\alpha-1} u(t, s)(f(s, \widehat{u}(s))-f(s, u(s))) d s\right| \\
\leqslant & \frac{M_{1}}{\Gamma(\gamma)}\left(\left|\widehat{u}_{0}-u_{0}\right|+L_{3}\|\widehat{u}-u\|_{c_{1-\gamma}}\right)+\frac{M_{1} L_{1}}{\Gamma(\alpha)} \int_{0}^{t}(t-s)^{\alpha-1}|\widehat{u}(s)-u(s)| d s \\
\leqslant & \frac{M_{1}}{\Gamma(\gamma)}\left(\left|\widehat{u}_{0}-u_{0}\right|+L_{3}\|\widehat{u}-u\|_{c_{1-\gamma}}\right)+\frac{M_{1} L_{1} t^{1-\gamma}}{\Gamma(\alpha)} \int_{0}^{t}(t-s)^{\alpha-1}|\widehat{u}(s)-u(s)| d s \\
& \leqslant \frac{M_{1}}{\Gamma(\gamma)}\left(\left|\widehat{u}_{0}-u_{0}\right|+L_{3}\|\widehat{u}-u\|_{c_{1-\gamma}}\right)+\frac{M_{1} L_{1} T^{1-\gamma+\alpha}}{\Gamma(\alpha+1)}\|\widehat{u}-u\|_{c_{1-\gamma}}
\end{aligned}
$$

which implies that

$$
\|\widehat{u}-u\|_{c_{1-\gamma}} \leqslant \frac{\frac{M_{1}}{\Gamma(\gamma)}}{1-M_{1}\left(\frac{L_{3}}{\Gamma(\gamma)}+\frac{L_{1} T^{1-\gamma+\alpha}}{\Gamma(\alpha+1)}\right)}\left|\widehat{u}_{0}-u_{0}\right| .
$$

Hence, by the inequality (3.1) we conclude that the solution $u$ of the problem (1.1) is continuously dependent on the initial data $\mathfrak{u}_{0}$. This completes the proof.

\section{Acknowledgment}

I would like to thank the anonymous referee for his/her efforts in reading the paper and his/her valuable comments.

\section{References}

[1] S. Abbas, M. Benchohra, M. Bohner, Weak Solutions for Implicit Differential Equations with Hilfer-Hadamard Fractional Derivative, Adv. Dyn. Syst. Appl., 12 (2017), 1-16. 1

[2] S. Abbas, M. Benchohra, J.-E. Lazreg, Y. Zhou, A survey on Hadamard and Hilfer fractional differential equations: Analysis and stability, Chaos Solitons Fractals, 102 (2017), 47-71. 1

[3] M. Bragdi, M. Hazi, Existence and controllability results for an evolution fractional integrodifferential systems, Int. J. Contemp. Math. Sci., 5 (2010), 901-910. 2

[4] A. Debbouche, V. Antonov, Approximate controllability of semilinear Hilfer fractional differential inclusions with impulsive control inclusion conditions in Banach spaces, Chaos Solitons Fractals, 102 (2017), 140-148. 1

[5] D. B. Dhaigude, S. P. Bhairat, Existence and uniqueness of solution of Cauchy-type problem for Hilfer fractional differential equations, Comm. Appl. Anal., 22 (2018), 121-134. 1

[6] W. E. Fitzgibbon, Semilinear functional differential equations in Banach space, J. Differential Equations, 29 (1978), 1-14. 2.4

[7] A. Friedman, Partial Differential Equations, Holt, Rinehat and Winston, New York, (1969). 
[8] X. L. Fu, X. B. Liu, Existence of periodic solutions for abstract neutral non-autonomous equations with infinite delay, J. Math. Anal. Appl., 325 (2007), 249-267. 2.4

[9] K. M. Furati, M. D. Kassim, N. e-. Tatar, Existence and uniqueness for a problem involving Hilfer fractional derivative, Comput. Math. Appl., 64 (2012), 1616-1626. 1, 2, 2

[10] H. Gou, B. Li, Existence of mild solutions for fractional nonautonomous evolution equations of Sobolev type with delay, J. Inequal. Appl., 2017 (2017), 20 pages. 1

[11] A. Granas, J. Dugundji, Fixed Point Theory, Springer-Verlag, New York, (2003). 2.6

[12] H. Gu, J. J. Trujillo, Existence of mild solution for evolution equation with Hilfer fractional derivative, Appl. Math. Comput., 257 (2015), 344-354. 1

[13] R. Hilfer (ed.), Applications of Fractional Calculus in Physics, World Sci. Publ., River Edge, (2000). 1, 2

[14] R. Hilfer, Fractional calculus and regular variation in thermodynamics, World Sci. Publ., River Edge, (2000).

[15] R. Hilfer, Fractional time evolution, World Sci. Publ., River Edge, (2000). 1

[16] R. Kamocki, C. Obczynski, On fractional Cauchy-type problems containing Hilfer's derivative, Electron. J. Qual. Theory Differ. Equ., 50 (2016), 12 pages. 1

[17] A. A. Kilbas, H. M. Srivastava, J. J. Trujillo, Theory and applications of fractional differential equations, Elsevier Science B.V., Amsterdam, (2006). 2

[18] F. Li, Mild solutions for fractional differential equations with nonlocal conditions, Adv. Difference Equ., 2010 (2010), 9 pages. 2

[19] N. I. Mahmudov, M. A. McKibben, On the Approximate Controllability of Fractional Evolution Equations with Generalized Riemann-Liouville Fractional Derivative, J. Funct. Spaces, 2015 (2015), 9 pages. 1

[20] Z.-D. Mei, J.-G. Peng, J.-H. Gao, Existence and uniqueness of solutions for nonlinear general fractional differential equations in Banach spaces, Indag. Math. (N.S.), 26 (2015), 669-678. 1

[21] Z.-D. Mei, J.-G. Peng, J.-H. Gao, General fractional differential equations of order $\alpha \in(1,2)$ and Type $\beta \in[0,1]$ in Banach spaces, Semigroup Forum, 94 (2017), 712-737. 1

[22] G. M. Mophou, G. M. N'Guérékata, Mild solutions for semilinear fractional differential equations, Electron. J. Differential Equations, 21 (2009), 1-9. 2

[23] A. Pazy, Semigroup of linear operators and applications to partial differential equations, Springer-Verlag, New York, (1983). 2.4

[24] Z̆. Tomovski, R. Hilfer, H. M. Srivastava, Fractional and operational calculus with generalized fractional derivative operators and Mittag-Leffler type functions, Integral Transforms Spec. Funct., 21 (2010), 797-814. 2

[25] D. Vivek, K. Kanagarajan, E. M. Elsayed, Some Existence and Stability Results for Hilfer-fractional Implicit Differential Equations with Nonlocal Conditions, Mediterr. J. Math., 15 (2018), 21 pages. 1

[26] D. Vivek, K. Kanagarajan, S. Sivasundaram, Dynamics and Stability Results for Hilfer Fractional Type Thermistor Problem, Fractal Fractional, 2017 (2017), 80-93. 1

[27] J. R. Wang, Y. Zhang, Nonlocal initial value problems for differential equations with Hilfer fractional derivative, Appl. Math. Comput., 266 (2015), 850-859. 1

[28] M. Yang, Q.-R. Wang, Approximate controllability of Hilfer fractional differential inclusions with nonlocal conditions, Math. Methods Appl. Sci., 40 (2017), 1126-1138. 1

[29] H. P. Ye, J. M. Gao, Y. S. Ding, A generalized Gronwall inequality and its application to a fractional differential equation, J. Math. Anal. Appl., 328 (2007), 1075-1081. 1, 4, 4 